\title{
PORTEFEUILLES VIRTUELS ET SIMULATION BOURSIÈRE : UNE APPROCHE LUDIQUE ET INTERACTIVE DU CAPITALISME
}

\author{
Jean-Thierry JULIA ${ }^{1}$
}

Les sites consacrés à la Bourse - ou à proprement parler à la transaction boursière - se sont révélés durant les dernières années comme des destinations prisées à la navigation de certaines catégories d'internautes. Avec notamment l'avènement du web, la Bourse aura troqué l'image d'une institution lointaine pour seuls notables et spécialistes de la finance, pour celle banalisée, liée à la pratique du petit porteur gérant son patrimoine en quelques clics de souris, entre courrier électronique et DVD.

S'inscrivant dans le cadre de recherches plus générales sur les NTIC et plus particulièrement sur la notion d'interactivité, la réflexion que nous proposons ici saisira le site boursier comme prétexte. L'examen d'un tel dispositif de médiation boursière le révèle en effet tout à fait caractéristique des potentialités qu'offre le multimédia interactif d'un point de vue communicationnel. Nous exposerons en premier lieu les spécifités de quelques sites consacrés à la bourse ; nous pourrons alors successivement reconnaître en ceux-ci, une illustration supplémentaire et tout à fait typique de nouvelles modalités de communication mises en évidence plus généralement au creux des dispositifs multimédias et

1 LERASS, Université Paul Sabatier-Toulouse 3

Recherches en communication, $\mathrm{n}^{\circ} 23$ (2005). 
interactifs, et que nous expliciterons ensuite en correspondance à nos observations. Nous envisagerons enfin ce qui pourra notamment se jouer d'un point de vue plus cognitif, dans le domaine de savoirs liés à la bourse et de façon corollaire à l'économie, dans le cadre d'une telle médiation revisitée par l'outil technologique.

\section{Bourse et internet}

La consultation de différents sites boursiers - retenus non par souci d'une quelconque représentativité mais sur la seule base de renseignements obtenus de plusieurs utilisateurs assidus ${ }^{1}$ - aura été à la base de cette réflexion. En matière de transaction boursière, de nombreux sites existent, qui ont vu le jour dès l'avènement d'internet, voire en France celui de la télématique et de l'usage minitel. Conformément aux rapidité et volumes croissants qu'ont connu la sphère des transactions de capitaux, l'implantation se tels services sur internet a encore été concomitant d'une ouverture de plus en plus large du secteur à ce qu'il est convenu d'appeler les «petits porteurs ». Le service web, mis en place par un acteur intermédiaire entre Bourse et particulier, qu'il soit courtier, banque ou société d'assurance, a contribué à un nouveau paysage boursier et notamment participé pour ce qui concerne ce segment du public, au l'usage des services de communication en ligne. Le fonctionnement de tels sites réside sur le principe d'une gestion de portefeuilles personnalisée, qui pourra avoir lieu en temps réel, quand ce paramètre temporel, pour des raisons liées à la fluctuation des marchés, pourra revêtir quelque importance dans le secteur qui nous intéresse. Dans un tel contexte technologique, l'utilisateur, actionnaire d'un nouveau type, peut se connecter à toute heure au service dont il est client attitré, et moyennant sa stricte identification afin d'accéder à ses avoirs, transmettre ses ordres d'achat, de vente, qui seront globalisés, compensés et

1 Nous donnons ici les références des sites qui auront peu ou prou retenu notre attention à l'occasion de cette réflexion. Comme c'est souvent le cas sur le web - le champ des sites boursiers ne contrevient pas à l'observation - les références et contenus de produits sont très volatiles et susceptibles de modifications (intégration à d'autres services, rachat de produits entre courtiers, fusion d'établissements bancaires...). La liste proposée a été établie en juin 2001. Mise à jour par la suite, il se peut néanmoins qu'à la suite de notre transcription, un certain nombre de références soient inopérantes ou aient changé de contenu : www.ingdirect.fr, $w w w$. boursedirect.com, www.consors.fr, www.cortal.fr, www.fimatex. $f r$, www.ibourse.fr, www.mesactions.fr, www.richelieufinance. $f r, w w w . s e l f t r a d e . f r, w w w . w a r g n y . f r$ 
répercutés automatiquement sur le marché financier. Une fois au cœur du service, les interfaces peuvent changer, mais relèvent toujours de même fonctionnalités : affichage choisi ou personnalisé d'un certain nombre d'informations boursières, du plus modeste jusqu'à de véritables tableaux de bord financiers (valeurs du portefeuille, indices synthétiques, tendances internationales, indicateurs monétaires, etc.) ; parcours possibles au fil de multiples informations organisées sous la forme de différents écrans; et possibilité in fine pour l'utilisateur de prendre la main pour effectuer et transmettre, une fois sa décision suffisamment informée, un ou plusieurs ordres de transaction.

Jusque-là, le dispositif évoqué relève de fonctionnalités classiques dans le domaine des NTIC. Il s'agit en effet pour l'essentiel, de stricts affichages d'informations en ligne, aussi complexes soient-ils. La quantité d'informations, et surtout le caractère d'instantanéité qui est ici fondamental, renvoient en effet aux deux grandes spécificités de la consultation de tels services : volume d'informations - non pas tant d'un point de vue technique comme ce serait le cas pour la consultation d'images, les informations transmises, données chiffrées pour l'essentiel, renvoyant ici à la catégorie textuelle ; consultation en ligne et donc effective en temps réel, quand le service pourra se conformer au caractère éphémère de telles informations et restituer dans l'instant - ce sera là son avantage - les fluctuations intrinsèques des mécanismes boursiers. Confronté à un ensemble d'informations conséquent, l'utilisateur se voit offrir par le dispositif, et ce de façon inédite en comparaison à d'autres médias, la possibilité de consultations personnalisées, aussi bien quant à leur objet (l'utilisateur s'oriente vers l'affichage d'informations de son choix), que quant à leur rythme (il procède à ces choix selon un rythme qui ne relève que de sa convenance). Nous reprenons plus généralement dans ce qui suit, ce qui pourra constituer une première dimension de la notion d'interactivité, sous-couvert de laquelle le dispositif web, et plus largement celui interactif, autorisera de façon inédite ce qui dès lors est entendu par le vocable de «parcours multiples ».

\section{Consultation interactive}

Resituons tout d' abord rapidement le dispositif NTIC - ou dispositif multimédia interactif - dans la filiation de technologies informatiques. Dans la convergence de techniques observée autour de ces «médias 
électroniques », outre télécommunications et audiovisuel, l'informatique viendra imprégner de façon notable l'ensemble des dispositifs issus de cette rencontre. La technique informatique, étymologiquement celle du traitement automatique de l'information, a de tout temps envisagé l'interlocuteur humain comme fournisseur de cette information à traiter. Dans les médias qui nous intéressent, si l'interlocuteur devient le « correspondant à distance » du monde des télécommunications, ou encore le «spectateur » d'une société de l'image, il n'en est pas moins un utilisateur et un fournisseur - ne serait-ce qu'a minima - d'informations au dispositif. L'informatique a ainsi contribué à discerner dans les architectures techniques proposées, les « périphériques d'entrée », spécifiques de ce que l'utilisateur, sollicité, vient par sa contribution alimenter le dispositif technique en informations. Le «média électronique » prend ainsi mesure de l'activité de production informationnelle de l'utilisateur, inaugurant la notion d'interactivité qui en découle. Il ne s'agira alors ici pas tant d'examiner la dimension technique du média, que celle, du côté de l'utilisateur, liée à l'avènement de nouvelles modalités de fourniture et d'accès à l'information. CD-ROM, bornes interactives, sites web - et ceux dédiés à la transaction boursière n'en sont qu'un cas particulier - cristalliseront autour d'une même caractéristique interactive ce qui pourra se révéler une nouvelle posture de l'utilisateur.

Interrogée du point de vue même du dispositif interactif, la notion d'interactivité, ramène inévitablement à l'étude des «modalités de production » des messages notamment constitutifs de ce type de document ${ }^{1}$. Il convient ici d'entrevoir du côté des théories linguistiques de l'énonciation, les modalités de production et de réalisation de tels énoncés. Une interaction se précise en effet dans la possibilité pour l'utilisateur d'exercer certaines compétences pour l'énonciation, qui est, à l'inverse de son résultat en tant que tel, le processus lui-même de production - ou tout du moins nous le verrons plus loin d'instanciation ou d'actualisation - de ce contenu ou énoncé. L'interactivité est notamment avérée quand l'utilisateur et le support technique pour la présentation du document se voient associés à la réalisation effective du message, à l'actualisation de l'énoncé. Ils coopèrent alors dans

1 V. MABILLOT, Mises en scènes de l'interactivité. Représentations des utilisateurs dans les dispositifs de médiations interactives, thèse de doctorat en Sciences de l'information et de la communication, Université Lyon II, 2000. 
l'acte d'énonciation qui révèlera à un niveau élémentaire un contenu, et partant, plus globalement, autorisera l'accès à l'ensemble d'un document. Dans de tels dispositifs et documents interactifs, les énoncés non encore actualisés demeurent latents ; c'est par ses actions que l'utilisateur va réunir les conditions de leur énonciation et contribuer ainsi, si ce n'est à leur « réalisation », tout du moins à leur instanciation. En amont de l'énonciation, et dès la conception de ce type de document, les pré-énoncés s'insèrent néanmoins déjà dans une structure discursive, pour l'instant incomplète ( $c f$. fig. 1). Il conviendra pour l'utilisateur, lors de sa confrontation au document global, de compléter celui-ci par un certain nombre de ses actions. Quand de telles actions, opératoires, viendront ainsi autoriser l'accès à certaines sous-parties du document, le dispositif dans son ensemble pourra simuler les modalités de l'échange au fondement de la communication.

Fig. 1 - Enonciation et pré-énonciation

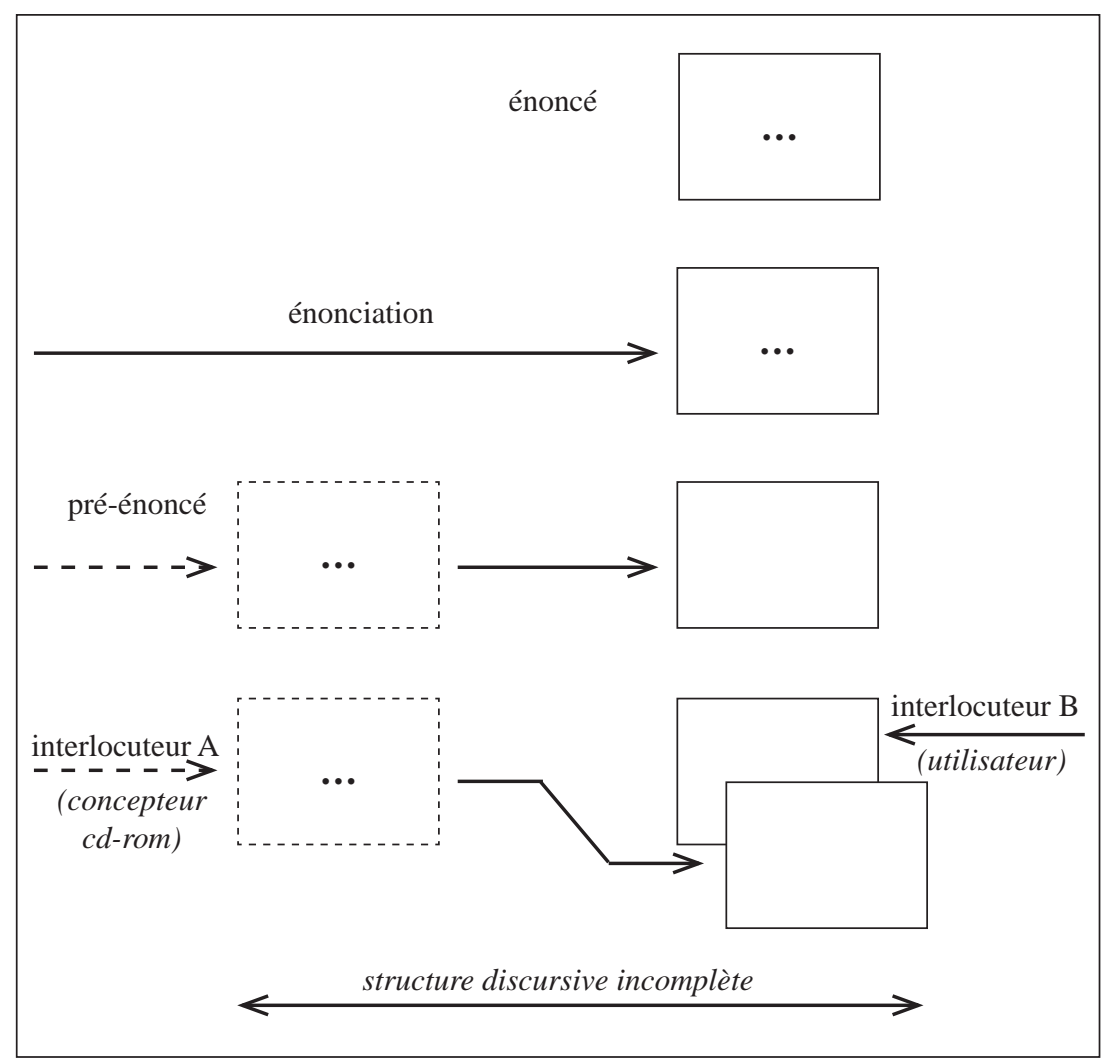


Dans une première étape de l'histoire de l'écriture interactive, ce ne sont pas tant des contenus inédits que les «parcours multiples » au sein de ces contenus, qui seront révélés par la confrontation aux documents de différents utilisateurs, ou lors d'utilisations successives d'un même utilisateur. La bifurcation dans les parcours de consultation, la cascade de ses choix, sont synonymes de modifications et d'actualisation du récit, récit dans le déroulement duquel l'utilisateur est alors partie prenante. Mais le nombre n'induit en rien la nature de l'échange qui devait faire advenir la machine au rang de véritable partenaire dans le dialogue. Conviendra-t-on ici tout au plus qu'en place d'interactivité, nous n'avions affaire qu'à une simple réactivité ${ }^{1}$.

\section{Simulation bousière}

Au-delà de cette première dimension d'interactivité dans la consultation, une véritable interaction se noue autour du dispositif boursier en ligne, à l'occasion de la transmission d'ordre. Elle est implémentée sous la forme désormais classique de «formulaire» (ou analogue), ou bien encore plus simplement sous forme de courrier électronique. Nous parlerons ici de véritable interaction, puisque, au terme et après globalisation des transactions recueillies sur le site, ce ne sera plus une action en réponse automatique qui sera mise en œuvre, mais bien une opération de type financier. Dans l'enceinte de la Bourse, sera lancé l'acte d'achat ou de vente, qui dépasse alors le cadre de notre étude liée aux NTIC. Nous ne nous appesantirons alors pas davantage sur cette interaction effective, quand elle est notamment conforme à celles qui succèdent à l'entrevue avec le banquier, le courtier, ou à la transmission d'ordre par écrit. Notons simplement que l'outil informatique intervient là encore dans de telles opérations : bon nombre de transactions se font aujourd'hui par voie électronique, et reprennent, à un autre niveau, de semblables modalités d'achat et de vente entre interlocuteurs professionnels à celles jusqu'ici décrites pour le petit porteur en ligne. Un marché - fût-il financier - n'est autre que la résultante de telles interactions, au-delà d'une simple interactivité machinique.

1 F. SÉGUY, Les produits interactifs et multimédias. Méthodologies, conception, écritures, Grenoble, PUG, 1999. 
Nous voudrions maintenant nous attacher à ce qui motivait effectivement la présente réflexion, autour de la conjugaison de facteurs liés à la Bourse et d'autres plus spécifiquement aux NTIC.

Nous avons en effet pu observer sur plusieurs de ces services en ligne, outre les services d'information et de transmission d'ordre, la possibilité offerte à l'interlocuteur en ligne, d'immédiates mises en œuvre de décisions boursières, pour l'instant non effectives, construites autour de transactions et opérations - n'ayant jusque-là que valeur d'essai ou de test - et étayées par de nombreuses simulations autour des paramètres du portefeuille de l'apprenti actionnaire en ligne. Quand les tableaux de bords ne reflétaient précédemment que la réalité économique et financière extérieure, ils vont être ici susceptibles d'intégrer un certain nombre de paramètres laissés à l'initiative de l'utilisateur, et qui concernent les transactions possibles. Ainsi pourra-t-il dans l'instant voir apparaître à l'écran la simulation et l'évolution à terme de son portefeuille, selon l'option d'achat ou de vente dont il pourrait donner ordre. Les rubriques de ce type sont plus ou moins conséquentes au sein des sites étudiés. Elles seront présentes a minima dans le cas d'un simple calcul prévisionnel des gains liés à la détention d'obligations ou de produits financiers composites, assurés de retour de dividendes. Plus complexe sera l'écran pour une totale simulation : sur la base du portefeuille choisi, et outre les intérêts, il répercutera dans la durée non seulement les conséquences des décisions en matière d'achat ou de vente, mais encore celles moins déterministes liées aux variations - prévues, prévisibles, possibles - de différents indicateurs boursiers que l'utilisateur choisit de convoquer. Au-delà de l'outil de suivi quotidien et en temps réel de d'un portefeuille que nous évoquions plus haut, l'utilisateur du site boursier a ici loisir, comme le vantent les enseignes proposant ce service, de mettre en œuvre un véritable portefeuille virtuel. Il pourra être un outil pertinent pour la simulation et le suivi de valeurs, de façon à pouvoir « s'exercer avant d'agir ».

Nous resituerons ici un autre type d'interactivité, qui n'est plus simple réactivité de l'utilisateur qui consulte et fait défiler un certain nombre d'écrans, mais interactivité autonome selon J.-P. Balpe ${ }^{1}$. Elle n'est ainsi envisageable qu'autour de documents, où, renvoyant à ce que nous mentionnons plus haut, les contenus ne sont plus uniquement

1 J.-P. BALPE, Techniques avancées pour l'hypertexte, Paris, Hermès, 1996. 
fournis par le simple dispositif technique, comme simples pré-énoncés. Dès que l'utilisateur quitte une position réactive et ne valide plus seulement un choix d'accès à des informations, son action accède alors au rang de contenu (ici texte et chiffres, mais ce pourrait être plus généralement des images, sons, scripts, etc.). Cette action contribue alors différemment à l'énoncé réalisé dans l'entre-deux de l'utilisateur et du dispositif : non plus modalité d'accès à des contenus déjà existants, le contenu produit est ici intrinsèquement le résultat commun des contributions de l'utilisateur et de celles du dispositif. Au final, le «document »-ici, l'état, après opérations et simulations, du portefeuille virtuel - change potentiellement de statut : il se construit à l'image d'un « document ouvert » qui pourra être modifié et recréé, recalculé, à partir de diverses contributions de l'utilisateur.

\section{Médiation interactive}

Une telle interactivité, entre utilisateur et dispositif technique, pourra encore trouver dans le champ de la linguistique pragmatique, les notions adéquates à son analyse. Selon le modèle de l'échange discursif de l'Ecole genevoise ${ }^{1}$, la forme monologale ou dialogale d'un échange (le nombre d'interlocuteurs), se double en effet d'une autre fonction qui caractérise un discours de monologique ou dialogique : l'échange, constitutif du dialogue, alterne interventions initiative et réactive des interlocuteurs, autour d'une fonction illocutoire que l'une impose à l'autre, indépendamment du contenu. Ainsi, une demande de réponse, sans même être mentionnée explicitement dans les termes employés, n'en est pas moins signifiée implicitement lors d'une question, d'une offre, etc. ( $c f$. fig. 2). Le « dialogue » avec le média interactif ne s'avèrera ainsi qu'un discours certes dialogal, mais néanmoins monologique, où l'utilisateur humain n'a d'autres possibilités que de tenir la position réactive.

1 E. ROULET et al., L'articulation du discours en français contemporain, Berne, Peter Lang, 1985. 
fig. 2 - Formes de l'échange et fonctions illocutoires

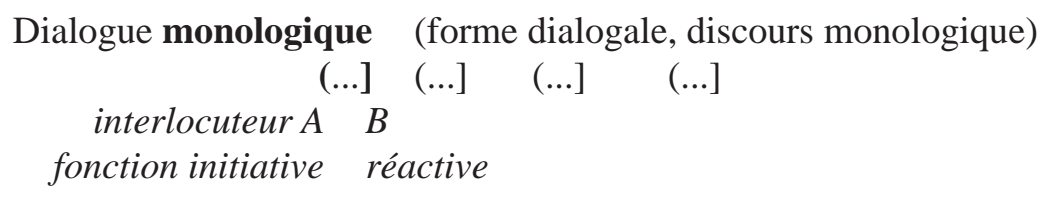

Monologue dialogique (forme monologale, discours dialogique)

$\begin{array}{cl}(\ldots) \quad(. . & (\ldots) \quad . .) \\ \text { initiative } & \text { (re-)initiative } \\ & \text { de } A\end{array}$

Dialogue dialogique (forme dialogale, discours dialogique)

$$
\begin{aligned}
& (\ldots] \quad(. . \quad[\ldots) \quad \text {...] } \quad(. . .] \\
& \text { initiative (re-)initiative } \\
& \text { de A de B }
\end{aligned}
$$

Une telle modalité au sein de la médiation technique, inédite dans le monde des médias, correspond bien avec celle que nous observions en matière de simulation, notamment boursière. A l'occasion de telles fonctionnalités, le dispositif se révèle dialogique, au bénéfice de l'utilisateur en ligne (ou encore hors ligne avec le cas du CD-ROM), lequel accède alors au rang de « réel » interlocuteur dans l'échange, tout du moins du point de vue linguistique évoqué.

Destinés in fine à la transaction boursière, les sites mentionnés n'en véhiculent pas moins certains savoirs sur les mécanismes, l'institution et la pratique boursiers. Ces savoirs ne sont plus seulement savoirs assertés, simples présentation ou exposition de la chose (comme l'observation directe de l'activité en Bourse), non plus seulement savoirs articulés au sein d'un discours qui viserait à leurs explicitation et transmission par le biais symbolique (comme pourraient les reprendre un enseignement académique ou une émission télévisée), mais savoirs - ou collection de savoirs et savoir-faire parcellaires - liés à d'immédiates mises en œuvre, construits par manipulation de portefeuilles, étayés par simulations diverses et variées : savoir «démontré», issu de ces nombreuses démonstrations. Un tel dispositif de simulation et de manipulation pourra ainsi contribuer à l'appréhension cognitive de 
l'objet. Dépassant le premier type d'interactivité observé précédemment, un second est alors envisagé, qui sera constitutivement lié aux simulations.

Au-delà de simples facilitations de la consultation d'un objet, il pourra en démultiplier la perception et la compréhension : augmenter la valeur initiale et observer l'évolution des dividendes, faire varier les indicateurs externes et apprécier leurs conséquences sur son propre pécule. Dans le prolongement d'autres exemples que l'on trouvera plus aisément dans le domaine visuel (faire tourner l'image et ainsi observer la sculpture ; crayonner sur l'image et retrouver les lignes de force de la toile d'un maître, etc.), nous pourrions proposer dans une suite à cette réflexion, de généraliser, sur la base d'une telle interactivité, et quels qu'en soient ses domaines d'application, la notion d' «image actée » selon Weissberg ${ }^{1}$ à celle plus globale d' « objet acté ». Et nous rejoindrions encore Jean-Louis Boissier, concepteur-réalisateur multimédia, qui suggère une approche renouvelée de ce que l'on pourrait entendre par interactivité : "L'interactivité n'est-elle pas ce qui simule des interactions réelles ? $»^{2}$.

Dans de possibles réitérations, toujours à l'initiative de l'utilisateur - et qui seraient plus délicates en situation de co-présence par égard à l'interlocuteur -, et dans le jeu qui dès lors s'instaure autour de ces multiples simulations, le dispositif interactif pourra encore instiller, une certaine dimension ludique, susceptible alors de quelque conséquence sur le plan cognitif. Comme le mentionne Karine Douplitzky, l'interactivité pourrait ainsi marquer le passage d'une conventionnelle relation face à un quelconque média, à une interaction plus ludique avec l'objet qu'il (re)-présente 3 .

Outre sur les sites boursiers, c'est en effet ce que l'on peut encore observer sous diverses formes dans les produits multimédias interactifs, depuis le plus modeste des roll-over («objet survolé » par la souris et qui se modifie à son passage) jusqu'aux complexités toujours grandissantes des jeux vidéos, qui quant à eux mettent en œuvre de nombreuses

1 J.-L. WEISSBERG, Présences à distance, Paris, L'Harmattan, 1999.

2 J.-L. BOISSIER, «Le moment interactif », Moments de Jean-Jacques Rousseau. Confessions et Rêveries, Paris, Gallimard multimédia, 2000, CD-ROM (livret).

3 K. DOUPLITZKY, « Peut-on pleurer devant un cd-rom ? », Les cahiers de médiologie, $\mathrm{n}^{\circ}$ 1, Paris, Gallimard, 1996. 
contributions de l'utilisateur, tant dans leur nombre que dans leur variété. Ainsi, si le dispositif autorise désormais le faire lié à une dimension interactive, demeure-t-il néanmoins dans le registre d'une communication médiatisée, et lors dans le seul registre du «comme si ». Que le faire puisse se prolonger alors dans le «faire comme si » inhérent au jeu ${ }^{1}$ semblerait en définitive inscrit au fondement même du dispositif multimédia interactif.

Dans le prolongement du constat de Jean-Louis Boissier autour de la notion de perspective et dans le champ de l'esthétique ${ }^{2}$, la médiation boursière, au fil de sites dédiés à quelque simulation et au bénéfice de ce multimédia interactif, pourra aussi contribuer, au-delà de sa visibilité ou de sa lisibilité, en une plus grande jouabilité de la sphère capitaliste.

1 R. CAILlOIS, Les jeux et les hommes. Le masque et le vertige, Paris, Gallimard, 1958.

2 J.-L. BOISSIER, «La perspective interactive. Visibilité, lisibilité, jouabilité », Revue d'esthétique, n 39, Paris, éd. J.-M. PLACE, 2001. 
